\title{
Evaluation and Integration of Genetic Signature for Prediction Risk of Nasopharyngeal Carcinoma in Southern China
}

\author{
Xiuchan Guo, ${ }^{1,2,3}$ Cheryl A. Winkler, ${ }^{4}$ Ji Li, ${ }^{1}$ Li Guan, ${ }^{5}$ Minzhong Tang, ${ }^{6,7}$ \\ Jian Liao, ${ }^{8}$ Hong Deng, ${ }^{7}$ Guy de Thé, ${ }^{9}$ Yi Zeng, ${ }^{2,6}$ and Stephen J. O'Brien ${ }^{5}$ \\ ${ }^{1}$ Key Laboratory of Laboratory Medicine, School of Laboratory Medicine and Life Science, Wenzhou Medical University, \\ Wenzhou 325000, China \\ ${ }^{2}$ State Key Laboratory for Infectious Diseases Prevention and Control, Institute for Viral Disease Control and Prevention, \\ Chinese CDC, Beijing 10052, China \\ ${ }^{3}$ ICF International, Atlanta, GA 30329, USA \\ ${ }^{4}$ Basic Research Laboratory, Frederick National Laboratory, Leidos Biomedical Research, Inc., National Cancer Institute, \\ Frederick, MD 21702, USA \\ ${ }^{5}$ Theodosius Dobzhansky Center for Genome Bioinformatics, St. Petersburg State University, St. Petersburg 199004, Russia \\ ${ }^{6}$ College of Life Science and Bio-Engineering, Beijing University of Technology, Beijing 100022, China \\ ${ }^{7}$ Cancer Center, Wuzhou Red Cross Hospital, Guangxi 543002, China \\ ${ }^{8}$ Cangwu Institute for Nasopharyngeal Carcinoma Control and Prevention, Wuzhou, Guangxi 543100, China \\ ${ }^{9}$ Institut Pasteur, 75724 Paris, France
}

Correspondence should be addressed to Xiuchan Guo; xiuchan88@yahoo.com and Yi Zeng; zengy@public.bta.net.cn

Received 18 February 2014; Accepted 30 June 2014; Published 10 August 2014

Academic Editor: Urszula Demkow

Copyright (C) 2014 Xiuchan Guo et al. This is an open access article distributed under the Creative Commons Attribution License, which permits unrestricted use, distribution, and reproduction in any medium, provided the original work is properly cited.

\begin{abstract}
Genetic factors, as well as environmental factors, play a role in development of nasopharyngeal carcinoma (NPC). A number of single nucleotide polymorphisms (SNPs) have been reported to be associated with NPC. To confirm these genetic associations with NPC, two independent case-control studies from Southern China comprising 1166 NPC cases and 2340 controls were conducted. Seven SNPs in ITGA9 at 3p21.3 and 9 SNPs within the 6p21.3 HLA region were genotyped. To explore the potential clinical application of these genetic markers in NPC, we further evaluate the predictive/diagnostic role of significant SNPs by calculating the area under the curve (AUC). Results. The reported associations between ITGA9 variants and NPC were not replicated. Multiple loci of GABBR1, HLA-F, HLA-A, and HCG9 were statistically significant in both cohorts ( $P_{\text {combined }}$ range from $5.96 \times 10^{-17}$ to 0.02 ). We show for the first time that these factors influence NPC development independent of environmental risk factors. This study also indicated that the SNP alone cannot serve as a predictive/diagnostic marker for NPC. Integrating the most significant SNP with IgA antibodies status to EBV, which is presently used as screening/diagnostic marker for NPC in Chinese populations, did not improve the AUC estimate for diagnosis of NPC.
\end{abstract}

\section{Background}

Nasopharyngeal carcinoma (NPC) is rare in most regions of the world; however, it is a common cancer in Southern China, especially in the Guangdong and Guangxi Provinces. The incidence rate of NPC for males in the Southern Chinese provinces of Guangdong and Guangxi is more than 20 per 100,000 person-years and up to $25-40$ per 100,000 personyears in some areas bordering the Xijiang River and Pearl
River drainages in these two provinces $[1,2]$. It has been well established that Epstein-Barr virus (EBV) is strongly associated with NPC [3-5]. The association of EBV antibodies and NPC were first reported in 1966 [6]. Later, the presence of IgA antibodies to EBV in serum was found to serve as a predictive marker for NPC in Chinese populations [7-9]. IgA antibody titers to the EBV viral capsid antigen (EBV/IgA/VCA) and to the EBV early antigen (EBV/IgA/EA) have been used for the screening and diagnosis of NPC 
for many years in Southern Chinese populations [7, 9-11]. Epidemiological studies have pointed to other environmental factors (including consumption of salt-preserved fish, exposure to domestic wood-cooking fires, and exposure to occupational solvents) as having a role in development of NPC $[5,12]$.

Evidence for genetic modulation of NPC risk has accumulated recently. Familial aggregation of NPC cases has been observed in both high- and low-risk populations in different geographic regions [5, 13-15]. Several studies have shown associations between HLA genes and NPC [16-21]. The results from our phase I cohort confirm and extend previously reported HLA and NPC associations in Southern Chinese populations [22]. Two genome-wide association studies (GWAS) have identified multiple gene association with risk of NPC in Chinese ancestry cohorts [23, 24]. The first GWAS comprised 111 unrelated NPC cases and 260 controls and a replication sample set of 168 cases and 252 controls from the Malaysian Chinese population [23] reported evidence of association with ITGA9 on Chr 3p21.3121.2. The second GWAS was conducted in 277 Taiwanese NPC cases and 285 controls and included two independent replication sets. This group found associations with variants on Chr 6p21.3 in or near HCG9, HLA-A, HLA-F, and GABBR1 genes [24].

To investigate whether genetic variants can improve the EBV IgA antibodies test method for NPC diagnosis, we extended previously reported GWAS associations with NPC to Han Chinese from Southern China-the highest NPC incidence region. Here, two independent case-control studies were conducted-phase I cohort with 350 NPC cases and 619 controls and phase II cohort with 816 NPC cases and 1721 controls to determine if the polymorphisms of ITGA9, HLAA, HLA-F, GABBR1, and HCG9 were associated with NPC development or can be potential genetic markers for onset of NPC in a Southern Chinese population.

\section{Materials and Methods}

2.1. Cases and Controls (Table 1). The NPC cohorts were recruited from areas along the Xijiang River in Guangdong and Guangxi Provinces of Southern China in two collection phases $[5,25]$. Phase I participants were recruited from April 2000 to June 2001. NPC cases were either incident or prevalent biopsy-confirmed NPC cases. The controls were the case's spouse or geographically matched residents who were NPC-free at the time of study enrollment. Phase II study participants were recruited from November 2004 to October 2005. Cases were incident or prevalent, biopsy-confirmed NPC. Controls were NPC-free at the time of study enrollment and matched to NPC cases on age and district/township of residence. NPC cases were patients at Wuzhou Red Cross Hospital in Wuzhou City and outpatients at Cangwu Institute for NPC Control and Prevention in Cangwu County. All participants self-identified as Han Chinese and reported at least three generations of residency in Guangdong or Guangxi Province, China.
IgA antibodies to EBV capsid antigen (EBV/IgA/VCA) and $\operatorname{IgA}$ antibodies to EBV early antigen (EBV/IgA/EA) were determined by serological testing at the time of study enrollment. The cutoff titer for the seropositive status was at least $1: 10$ and 1:5 for IgA/VCA and IgA/EA, respectively, based on local standard. Blood samples were obtained from 350 NPC cases (66.6\% male) and 619 controls ( $42.8 \%$ male) for phase I; the mean age was 45 years \pm 11 and 46 years \pm 10 for NPC cases and controls, respectively. For phase II, blood samples were collected from 816 NPC cases ( $73.2 \%$ male) and 1721 controls ( $61.4 \%$ male); the mean age was 45 years \pm 11 and 46 years \pm 12 for NPC cases and controls, respectively. Family history of NPC, parental ancestry for three generations, dietary and smoking habits, household exposures to wood fires, and occupational exposures to solvents were also captured by questionnaire in the phase II cohort [5]. Participants were asked if there was a family history of NPC in first- (children, siblings, or parents), second- (aunts or uncles, nieces or nephews, and grandparents), or third-degree relatives (first cousins). Information was also collected on the frequency of consumption per month ( $\geq 3$ times/month, and $<3$ times/month) of salty fish and preserved meat. Questions on cigarette smoking included current and past smoking habits and number of cigarettes smoked per day. Questions on household and occupational exposures captured data on domestic exposure to wood fires for cooking and occupational exposures to solvents (e.g., formaldehyde, acetone, toluene, or xylene) and duration of exposure ( $>10$ years or $\leq 10$ years). Responses were recorded by double-entry and verification of all data was performed to avoid data entry errors. We excluded persons of minority ethnicity and those who had blood relatives in either the case or control group. We also did not allow overlap in participation between phase I and phase II; the cohorts were independent. Institutional review board approval was obtained from all participating institutions and informed consent was obtained from each study participant.

2.2. Genomic DNA Extraction. In phase I participants, DNA was extracted from whole blood or lymphoblastoid cell lines using QIAamp DNA blood maxi kit (Qiagen, Valencia, CA, catalog number 51194). More than $80 \%$ of the genotypes were determined from DNA directly extracted from whole blood. In phase II participants, DNA was extracted from whole blood by traditional phenol/chloroform method with Phase Lock Gel tube (Qiagen, MaXtract High Density, catalog number 129065).

2.3. Genotyping. In both phases I and II, 7 SNPs of ITGA9 on 3p21.3 and 9 SNPs within the GABBR1, HLA-F, HLA$A$, and HCG9 genes on chromosome 6 p21.3 were genotyped by using commercially available TaqMan SNP genotyping assays and GeneAmp PCR System 9700 (Applied Biosystems, Foster City, CA, USA), in accordance with the manufacturer's instructions. The sequence detection software was used for allelic discrimination. For quality control, 8 to 16 templatefree controls, one family sample [25], and $5 \%$ to $10 \%$ of duplicate samples were included in each 384-well plate. 
TABLE 1: Characteristics of participants in a study of nasopharyngeal carcinoma (NPC) in southern China.

\begin{tabular}{lcccc}
\hline & & Phase I & & Phase II \\
& Cases & Controls & Cases & $45 \pm 11.0(\mathrm{SD})$ \\
\hline Age (years) & $45 \pm 11.4(\mathrm{SD})$ & $46 \pm 9.7(\mathrm{SD})$ & $73.2(597 / 816)$ & $61.4(1056 / 1721)$ \\
Male, $\%$ & $66.6(233 / 350)$ & $42.8(265 / 619)$ & $95.8(782 / 816)$ & $42.3(731 / 1721)$ \\
IgA/VCA+ ${ }^{*}, \%$ & $95.4(334 / 350)$ & $44.7(277 / 619)$ & $72.4(591 / 816)$ & $2.6(44 / 1721)$ \\
IgA/EA+ ${ }^{*}, \%$ & $59.5(201 / 348)$ & $2.3(14 / 619)$ & 816 & 1721 \\
\hline Total & 350 & 619 & & \\
\hline
\end{tabular}

Age: the age at diagnosis of NPC for cases and age of enrollment for controls.

SD: standard deviation.

* Positive for IgA antibodies to Epstein-Barr virus capsid antigen.

${ }^{* *}$ Positive for IgA antibodies to Epstein-Barr virus early antigen.

The cutoff value for seropositive status is $1: 10$ for IgA/VCA and 1:5 for IgA/EA.

TABLE 2: Association between alleles of SNPs at 6 p21.3 and NPC in phase I and phase II combined.

\begin{tabular}{lccccccc}
\hline \multirow{2}{*}{ Gene-SNP } & \multirow{2}{*}{ Risk allele } & \multicolumn{2}{c}{ Phase I and phase II } & \multicolumn{2}{c}{ Phase I and phase II } & \multicolumn{2}{c}{ Phase II } \\
& & OR $(95 \% \mathrm{CI})^{*}$ & $P^{*}$ & OR $(95 \% \mathrm{CI})^{* *}$ & $P^{* *}$ & OR (95\% CI) $)^{* * *} P^{* * *}$ \\
\hline GABBR1-rs2267633 & $\mathrm{A}$ & $1.61(1.41-1.84)$ & $1.02 E-12$ & $1.48(1.17-1.87)$ & 0.001 & $1.41(1.02-1.95)$ & 0.03 \\
GABBR1-rs29230 & $\mathrm{T}$ & $1.64(1.45-1.89)$ & $1.36 E-13$ & $1.61(1.28-2.04)$ & $6.14 E-05$ & $1.61(1.16-2.22)$ & 0.004 \\
GABBR1-rs29232 & $\mathrm{A}$ & $1.35(1.21-1.49)$ & $1.85 E-08$ & $1.41(1.16-1.71)$ & 0.0006 & $1.33(1.01-1.76)$ & 0.05 \\
HLA-F-rs3129055 & G & $1.14(1.02-1.28)$ & 0.02 & $1.33(1.09-1.64)$ & 0.008 & $1.47(1.10-1.20)$ & 0.01 \\
HLA-A-rs2517713 & T & $1.61(1.43-1.82)$ & $2.44 E-16$ & $1.69(1.35-2.08)$ & $2.58 E-06$ & $1.64(1.20-2.22)$ & 0.003 \\
HCG9-rs9260734 & G & $1.67(1.47-1.87)$ & $5.96 E-17$ & $1.75(1.41-2.17)$ & $6.48 E-07$ & $1.75(1.28-2.44)$ & 0.0005 \\
HCG9-rs3869062 & A & $1.60(1.42-1.81)$ & $3.4 E-14$ & $1.63(1.30-2.04)$ & $1.97 E-05$ & $1.60(1.16-2.19)$ & 0.004 \\
HCG9-rs5009448 & C & $1.62(1.45-1.82)$ & $1.89 E-16$ & $1.66(1.33-2.06)$ & $3.46 E-06$ & $1.64(1.20-2.26)$ & 0.002 \\
HCG9-rs16896923 & T & $1.54(1.35-1.75)$ & $4.56 E-11$ & $1.69(1.33-2.13)$ & $2.19 E-05$ & $1.64(1.18-2.27)$ & 0.005 \\
\hline
\end{tabular}

OR: odds ratio. CI: confidence interval.

${ }^{*}$ Adjusted for sex and age.

** Additionally adjusted for EBV/IgA/VCA and EBV/IgA/EA titers.

*** Additionally adjusted for EBV/IgA/VCA and EBV/IgA/EA antibody titers and other environmental factors including family history with NPC, consumption of salt-preserved fish, exposure to domestic wood cooking fires, and exposure to occupational solvents.

2.4. Statistical Analysis. Hardy-Weinberg equilibrium (HWE) assumptions were independently tested for each SNP in cases and controls for each phase group as well as the two phases combined as a quality control measure. For allele association (Table 2, Supplementary Tables 1 and 2; Supplementary Material available online at http://dx.doi.org/ $10.1155 / 2014 / 434072$ ), the Armitage's trend test was used to calculate the $P$ value for additive allele effects on the disease penetrance. ORs were calculated by Mantel-Haenszel estimate based on contingency tables of allele-by-trait counts. For controlling the confounding covariates (age, sex, etc.), the stratified case-control test was performed. All results shown were adjusted for age and sex. In order to exclude the influence of EBV, we analyzed the associations between polymorphisms and the occurrence of NPC using EBV/IgA/VCA and $\mathrm{EBV} / \mathrm{IgA} / \mathrm{EA}$ antibody titers as covariates. For phase II, environmental factors including family history with NPC, consumption of salt-preserved fish, exposure to domestic wood-cooking fires, and exposure to occupational solvents were used as covariates. The receiver operator characteristic (ROC) curve was used to assess the diagnostic performance of EBV/IgA/VCA or EBV/IgA/EA alone, SNP alone, and the integration of these risk factors. Statistics were calculated in the statistical package SAS and SAS Genetics version 9.1.3.
Linkage disequilibrium (LD) maps, blocks, and haplotypes were generated by Haploview software [26].

\section{Results}

3.1. Association Results with SNPs on HLA Region at 6p21.3. As shown in Table 1, over 95\% of NPC cases (titer 1:10 to $1: 640$ ) and $42 \%-45 \%$ of the controls (titer $1: 10$ to $1: 160$ ) were positive for EBV/IgA/VCA antibodies; about $60 \%-$ $72 \%$ of NPC cases (titer $1: 5$ to $1: 640$ ) and $2 \%-3 \%$ of the controls (titer $1: 5$ to $1: 80$ ) were positive for $\mathrm{EBV} / \mathrm{IgA} / \mathrm{EA}$ antibodies in the two cohorts. EBV/IgA/EA positive serostatus was always concordant with IgA/VCA seropositive status. To replicate the results of the GWAS showing association between NPC and chromosome 6 [24], 9 SNPs within the HLA region previously found to be associated with NPC were genotyped (Table 2). The genotype frequencies for 9 polymorphisms conformed HWE expectations for two control groups; the call rate was $97.9 \%-99.3 \%$ for the 9 SNPs. Table 2 provides the risk alleles, the OR, 95\% confidence intervals (CIs), and $P$ values for phases I and II combined and phase II controlling for environmental factors. Eight SNPs in $G A B B R 1, H L A-A$, and HCG9 were significantly associated 


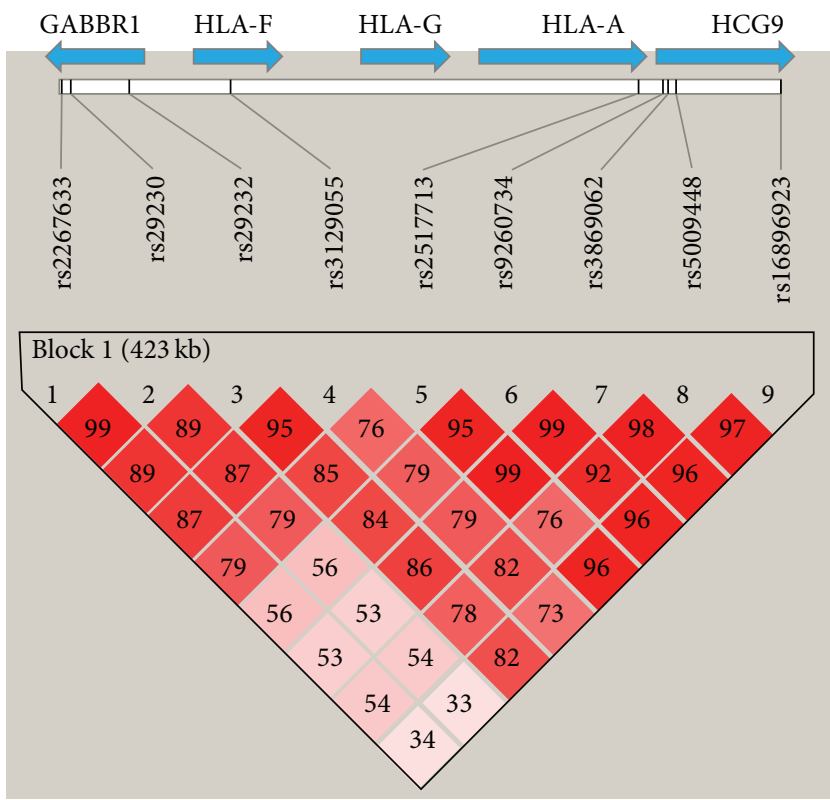

FIGURE 1: LD map based on $D^{\prime}$ was drawn using the genotype of the cases and controls.

with NPC in the phase I cohort (Supplementary Table 1: $P=0.0001-0.02$ ), phase II cohort (Supplementary Table 1: $\left.P=3.09 \times 10^{-12}-1.13 \times 10^{-6}\right)$, and combined phases I and II (Table 2: $P=5.96 \times 10^{-17}-1.85 \times 10^{-8}$ ). The SNP on HLA-F was significant in phase II and in the combined cohort but not in the smaller phase I cohort, although the ORs were in the same direction. After additionally controlling for EBV/IgA/VCA and EBV/IgA/EA antibody titers, 9 SNPs were also significant (Table 2). For the phase II cohort, after adjusting for sex, age, and EBV antibodies titers, we further controlled for environmental factors, which were shown to be associated with NPC in this cohort [5], including family history with NPC, consumption of salt-preserved fish, exposure to domestic wood-cooking fires, and exposure to occupational solvents as covariates. The 9 SNPs remained significantly associated with the risk of NPC (Table 2, last two columns).

Based on the LD map all 9 SNPs are in the same block (Figure 1). The HCG9-rs9260734, the most significant SNP, was used for evaluating whether the genetic signature can serve as a diagnostic marker for NPC. Table 3 presented the sensitivity, specificity, and accuracy for EBV/IgA/VCA, EBV/IgA/EA, and SNP test. The specificity (9.3\%) and accuracy (38\%) of HCG9-rs9260734 are lower than 50\%, which indicated that the SNP does not qualify as a diagnostic marker. To evaluate if the SNP can improve current IgA antibodies test for NPC diagnosis, we compared the receiver operating characteristic (ROC) curves between IgA/VCA or IgA/EA alone and integrated the SNP with status of IgA/VCA or IgA/EA. The area under the curve (AUC) for integrated markers did improve compared with IgA/VCA or IgA/EA alone $(0.917$ versus 0.915 for IgA/VCA; 0.833 versus 0.839 for IgA/EA; Figures 2(a) and 2(b)); however, this was not statistically significantly different.
TABLE 3: The diagnosis performance of IgA/VCA and IgA/EA and genetic signature.

\begin{tabular}{lccc}
\hline Test & Sensitivity (\%) & Specificity (\%) & Accuracy (\%) \\
\hline EBV/IgA/VCA & 95.7 & 56.9 & 69.8 \\
EBV/IgA/EA & 68.6 & 97.5 & 87.9 \\
HCG9-rs9260734 & 95.6 & 9.3 & 38.0 \\
\hline
\end{tabular}

Note: the threshold for IgA/VCA is $1: 10$ and for IgA/EA is $1: 5$.

3.2. Association Results with SNPs on ITGA9 at 3p21.3. To examine the influence of ITGA9 gene variants on NPC [23], 7 SNPs in ITGA9 were genotyped in phase I and phase II cohorts. Each of the 7 SNPs confirmed to HWE expectations in controls for both cohorts and the genotype call rate was $96.8 \%-99.4 \%$. No evidence of association was seen between 6 of the SNPs and NPC in phase I or phase II or in the combined analysis (Supplementary Tables 1 and 2). SNP (rs169111) was modestly significant in the combined analysis (Supplementary Table 2: OR $=1.4, P=0.03$ ) but not after adjusting for EBV IgA antibodies titers.

\section{Discussion}

In our study, we have demonstrated the strong associations of 9 SNPs located within GABBR1, HLA-F, HLA-A, and $H C G 9$ with NPC. Our results, from two independent Han Chinese NPC cohorts, confirm the previous associations and effect sizes reported in the Taiwanese GWAS [24]. The etiology of nasopharyngeal carcinoma is influenced by both genetic and environmental factors. EBV IgA antibody status is a strong predictive marker and plays an important role in NPC development in Southern Chinese populations [9, $10,27,28]$. Our results show that over $95 \%$ of NPC cases were $\mathrm{EBV} / \mathrm{IgA} / \mathrm{VCA}$ antibody positive and about $60 \%-72 \%$ of NPC cases were positive for EBV/IgA/EA antibodies. To exclude potential influence of EBV antibody status, we controlled EBV/IgA/VCA and EBV/IgA/EA antibody titers during the analysis. The results indicated that the variants of GABBR1, HLA-F, HLA-A, and HCG9 were still associated with NPC. In a previous study we reported that family history with NPC, consumption of salt-preserved fish, exposure to domestic wood-cooking fires, and exposure to occupational solvents were risk factors of NPC [5]. These environmental exposure data were available in our phase II cohort. When we adjusted for these factors in our association analysis, the 95\% confidence intervals overlapped indicating that variants on GABBR1, HLA-F, HLA-A, and HCG9 association were independent of environmental factors with NPC onset; the reduced statistical significance in the analysis adjusted for environmental factors reflects the small sample size with environmental data. These results, from two independent cohorts, affirm the associations of GABBR1, HLA-F, HLA-A, and HCG9 with NPC in the Han Chinese population. HLA$A$ and HCG9 have been reported to be associated with EBVpositive Hodgkin lymphoma and infectious mononucleosis caused by EBV infection $[29,30]$. This suggested that genetic variation chromosome $6 \mathrm{p} 21.3$ can influence the outcome of primary EBV infection and the level of viral persistence. 


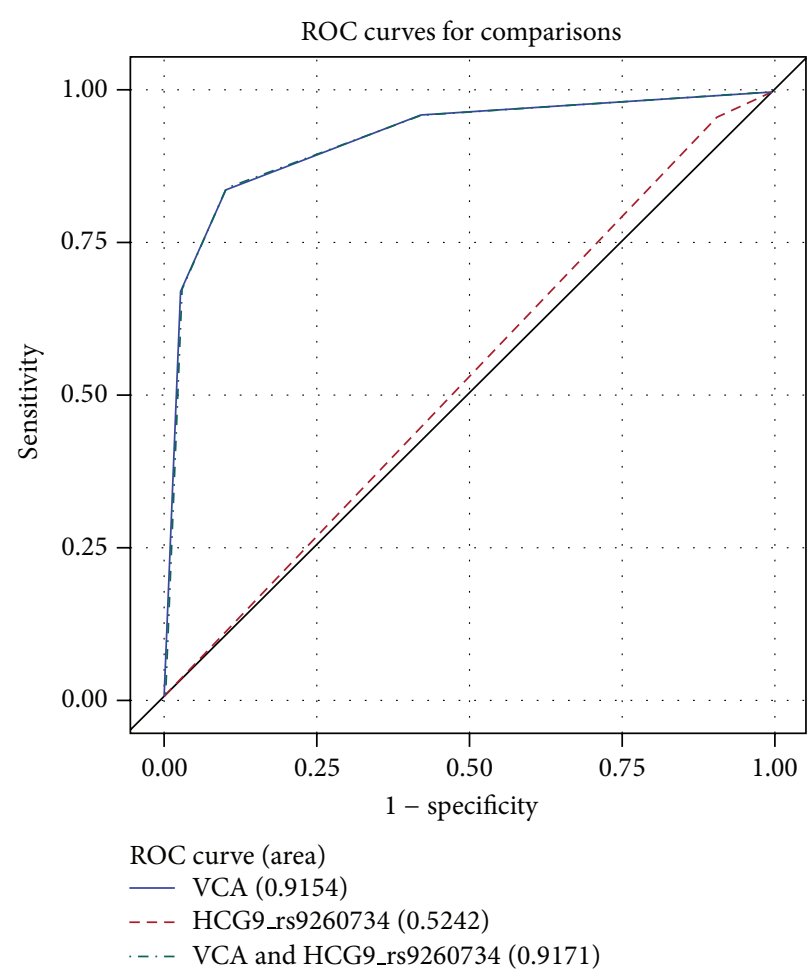

(a)

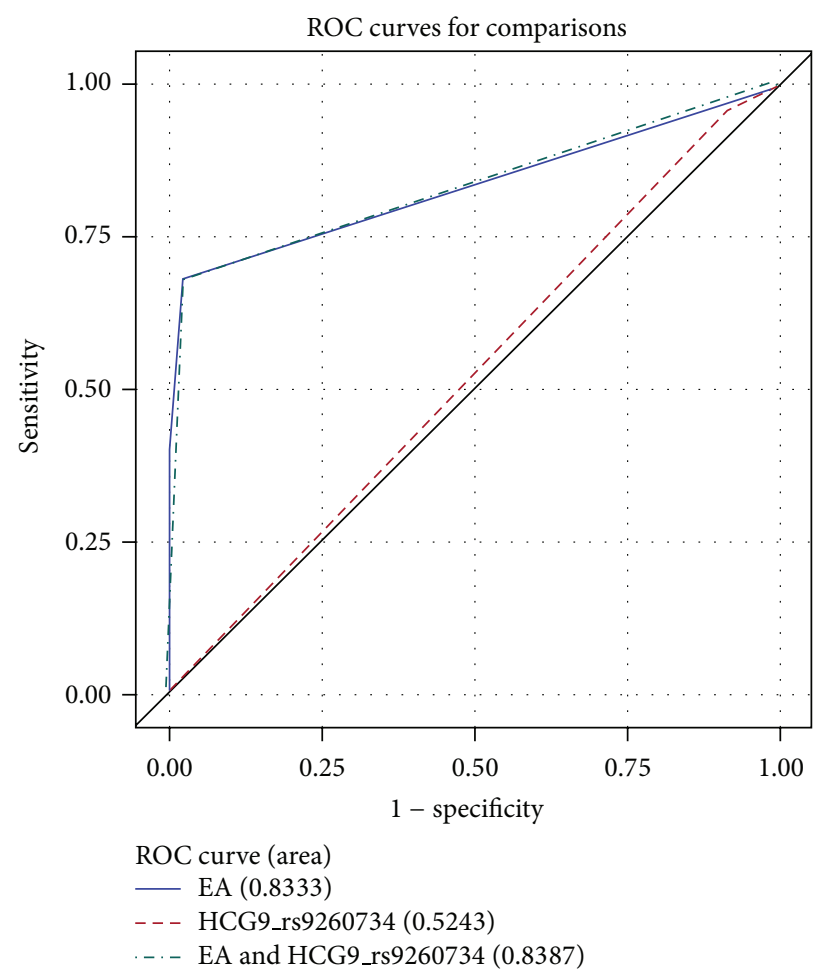

(b)

FIGURE 2: ROC curves.

A genome-wide expression profiling has revealed that increased EBV gene expression is strongly associated with inhibition of multiple HLA class I gene expression in NPC [31], further implicating these genes with NPC.

$\mathrm{EBV} / \mathrm{IgA} / \mathrm{VCA}$ and EBV/IgA/EA antibody titers, especially EBV/IgA/VCA, have been used for the screening and diagnosis of NPC for over 30 years in Southern Chinese populations [7, 9-11]. Along with the cancer genetic/genomic project development, new genetic variants associated with NPC are continuously being discovered. There is a growing need to evaluate the genetic markers for medical practice. Our interest is whether the significant SNPs can serve as a diagnostic marker or improve the IgA antibodies test for NPC prediction. Sensitivity, specificity, and accuracy are widely used statistics to quantify how good and reliable a test is. The receiver operating characteristic (ROC) curve is a graphic presentation of the relationship between both sensitivity and specificity and becomes the standard analytical tool for evaluating diagnostic tests. In this study, the specificity and accuracy of SNP was lower than 50\%; the AUC of SNP was less than 0.6 indicating SNP alone cannot be a diagnostic marker for NPC. Considering the positive rate for $\mathrm{EBV} / \mathrm{IgA} / \mathrm{VCA}$ antibodies in the general population is about $3 \%$ [10], we randomly selected samples and made the control group that contained 3\% IgA/VCA positive, then we repeated the ROC analysis; we obtained similar results. The AUC increased when IgA/VCA or IgA/EA was integrated with SNP; however, the effect was not statistically significant. To our knowledge, this is the first study to explore and incorporate the genetic variants to clinical use for NPC. Our results show that there is a strong association between variants of GABBR1, HLA-F, HLA-A, and HCG9 and NPC, but they cannot be useful for individualized risk prediction/diagnosis of NPC. However, risk profile based on a combination of genetic and other risk factors leads to an appreciable increased risk of disease and there is potential for increased predictive power as more genetic risk variants are detected [32].

We were unable to confirm the reported association with NPC of 7 SNPs in ITGA9 in our large Chinese Han population $(N=3506)$ from an NPC high incidence region of Southern China, although this study was well powered to detect a similar level of association. Based on our results, the minor allele frequencies (MAFs) for these 7 SNPs were lower (between 0.024 and 0.03). The previous study was carried out on a relatively small sample with 279 cases and 512 controls [23]; the few patients and a low MAF may have contributed to low statistical power for SNP association. However, two more recent NPC GWAS also failed to replicate the association of ITGA9 with NPC $[33,34]$.

Our study is unique in that two critical EBV antibody titers, as well as environmental factors, are available for inclusion in the statistical modeling. We controlled for these risk factors to determine the genetic association with NPC. This study systematically replicates the association from two NPC GWAS [23, 24] performed in NPC high-risk Asian populations. We also explored potential clinical use for significant genetic makers. Further study should focus on 
how these genetic variants impact function at the molecular and cellular levels to affect NPC development. Understanding the functional consequences of genetic variation will be critical to advancing our knowledge of the etiology of the disease and implementing rational medical strategies.

\section{Conclusion}

In summary, our results extend the association of the GABBR1, HLA-F, HLA-A, and HCG9 locus with NPC to the Han Chinese population of Southern China. We show for the first time that these factors are independent of environmental risk factors previously shown to influence NPC development. This study also indicated that EBV IgA antibodies in combination with these genetic makers may not be useful for diagnostic prognosis of NPC.

\section{Conflict of Interests}

No potential conflict of interests was disclosed.

\section{Acknowledgments}

This project has been funded in part with federal funds from the National Cancer Institute, National Institutes of Health, under contract HHSN26120080001E. This Research was supported (in part) by the Intramural Research Program of the NIH, National Cancer Institute, Center for Cancer Research. This research was supported in part by the National Natural Science Foundation of China, Grant no. 30672377 and Zhejiang Provincial Top Key Discipline of Laboratory Medicine, Key Science and Technology Innovation Team of Zhejiang Province (2010R50048). The authors thank all the participants in the cohorts. They thank Anna Satcher Johnson for reviewing and editing the paper; Xinjian Zhang, Randall Johnson, and James Lautenberger for statistical advice; and Michael Malasky and Mary J. McNally for excellent technical assistance. The content of this publication does not necessarily reflect the views or policies of the Department of Health and Human Services, and the mention of trade names, commercial products, or organizations does not imply endorsement by the US Government.

\section{References}

[1] J. J. Wu, H. Guo, and R. Su, "Analysis and forecast of incidence and mortality of nasopharynx cancer by time series in Zhongshan city," Chinese Journal of Hospital Statistics, vol. 8, no. 1, pp. $16-19,2001$.

[2] W. H. Jia, Q.-H. Huang, J. Liao et al., "Trends in incidence and mortality of nasopharyngeal carcinoma over a 20-25 year period (1978/1983-2002) in Sihui and Cangwu counties in southern China," BMC Cancer, vol. 6, article 178, 2006.

[3] J. Liu, L. Cassar, A. Pinto, and H. Li, "Mechanisms of cell immortalization mediated by EB viral activation of telomerase in nasopharyngeal carcinoma," Cell Research, vol. 16, no. 10, pp. 809-817, 2006.

[4] Y. G. Lee, Y. Hwang, K. Chen et al., "Effect of Epstein-Barr virus infection on global gene expression in nasopharyngeal carcinoma," Functional and Integrative Genomics, vol. 7, no. 1, pp. 79-93, 2007.

[5] X. Guo, R. C. Johnson, H. Deng et al., "Evaluation of nonviral risk factors for nasopharyngeal carcinoma in a high-risk population of southern China," International Journal of Cancer, vol. 124, no. 12, pp. 2942-2947, 2009.

[6] L. J. Old, E. A. Boyse, H. F. Oettgen et al., "Precipitating antibody in human serum to an antigen present in cultured burkitt's lymphoma cells," Proceedings of the National Academy of Sciences of the United States of America, vol. 56, no. 6, pp. 1699-1704, 1966.

[7] Y. Zeng, L. G. Zhang, H. Y. Li et al., "Serological mass survey for early detection of nasopharyngeal carcinoma in Wuzhou City, China," International Journal of Cancer, vol. 29, no. 2, pp. 139141, 1982.

[8] Y. Zeng, "Seroepidemiological studies on nasopharyngeal carcinoma in China," Advances in Cancer Research, vol. 44, pp. 121138, 1985.

[9] Y. Zeng and H. Deng, "A 10-year prospective study on nasopharyngeal carcinoma in Wuzhou City and Cangwu County," in The Epstein-Barr Virus and Associated Diseases, T. Tursz et al., Ed., pp. 735-741, Colloque INSERM/John Libbey Eurotext, 1993.

[10] H. Deng, Y. Zeng, Y. Lei et al., "Serological survey of nasopharyngeal carcinoma in 21 cities of south China," Chinese Medical Journal, vol. 108, no. 4, pp. 300-303, 1995.

[11] R. K. Y. Tsang, A. C. Vlantis, F. W. K. Ho, J. S. L. Tam, K. F. To, and C. A. Van Hasselt, "Sensitivity and specificity of Epstein-Barr virus IgA titer in the diagnosis of nasopharyngeal carcinoma: a three-year institutional review," Head and Neck, vol. 26, no. 7, pp. 598-602, 2004.

[12] E. T. Chang and H. Adami, "The enigmatic epidemiology of nasopharyngeal carcinoma," Cancer Epidemiology Biomarkers and Prevention, vol. 15, no. 10, pp. 1765-1777, 2006.

[13] T. M. Brown, C. W. Heath, R. M. Lang, S. K. Lee, and B. W. Whalley, "Nasopharyngeal cancer in Bermuda," Cancer, vol. 37, no. 3, pp. 1464-1468, 1976.

[14] C. M. Coffin, S. S. Rich, and L. P. Dehner, "Familial aggregation of nasopharyngeal carcinoma and other malignancies: a clinicopathologic description," Cancer, vol. 68, no. 6, pp. 1323-1328, 1991.

[15] K. S. Loh, B. C. Goh, J. Lu, W. Hsieh, and L. Tan, "Familial nasopharyngeal carcinoma in a cohort of 200 patients," Archives of Otolaryngology-Head and Neck Surgery, vol. 132, no. 1, pp. 82-85, 2006.

[16] S. Lu, N. E. Day, L. Degos et al., "Linkage of a nasopharyngeal carcinoma susceptibility locus to the HLA region," Nature, vol. 346, no. 6283, pp. 470-471, 1990.

[17] S. P. Hu, N. E. Day, D. R. Li et al., "Further evidence for an HLArelated recessive mutation in nasopharyngeal carcinoma among the Chinese," British Journal of Cancer, vol. 92, no. 5, pp. 967970, 2005.

[18] A. Hildesheim, R. J. Apple, C. Chen et al., "Association of HLA class I and II alleles and extended haplotypes with nasopharyngeal carcinoma in Taiwan," Journal of the National Cancer Institute, vol. 94, no. 23, pp. 1780-1789, 2002.

[19] M. J. Simons, G. B. Wee, S. H. Chan, K. Shanmugaratnam, N. E. Day, and G. de-Thé, "Immunogenetic aspects of nasopharyngeal carcinoma (NPC) III. HL-a type as a genetic marker of NPC predisposition to test the hypothesis that Epstein-Barr virus is an etiological factor in NPC," IARC Scientific Publications, vol. 11, part 2, pp. 249-258, 1975. 
[20] S.-B. Wu, S.-J. Hwang, A.-S. Chang et al., "Human leukocyte antigen (HLA) frequency among patients with nasopharyngeal carcinoma in Taiwan," Anticancer Research, vol. 9, no. 6, pp. 1649-1654, 1989.

[21] C. Lu, J. Chen, Y. Jin, H. Yang, S. Chan, and S. Tsai, "Genetic susceptibility to nasopharyngeal carcinoma within the HLA-A locus in Taiwanese," International Journal of Cancer, vol. 103, no. 6, pp. 745-751, 2003.

[22] M. Tang, Y. Zeng, A. Poisson et al., "Haplotype-dependent HLA susceptibility to nasopharyngeal carcinoma in a Southern Chinese population," Genes and Immunity, vol. 11, no. 4, pp. 334-342, 2010.

[23] C. C. Ng, P. Y. Yew, S. M. Puah et al., "A genome-wide association study identifies ITGA9 conferring risk of nasopharyngeal carcinoma," Journal of Human Genetics, vol. 54, no. 7, pp. 392397, 2009.

[24] K. Tse, W. Su, K. Chang et al., "Genome-wide association study reveals multiple nasopharyngeal carcinoma-associated loci within the HLA region at chromosome 6p21.3," The American Journal of Human Genetics, vol. 85, no. 2, pp. 194-203, 2009.

[25] X. C. Guo, K. Scott, Y. Liu et al., "Genetic factors leading to chronic Epstein-Barr virus infection and nasopharyngeal carcinoma in South East China: study design, methods and feasibility," Human Genomics, vol. 2, no. 6, pp. 365-375, 2006.

[26] J. C. Barrett, B. Fry, J. Maller, and M. J. Daly, "Haploview: analysis and visualization of LD and haplotype maps," Bioinformatics, vol. 21, no. 2, pp. 263-265, 2005.

[27] N. Raab-Traub, "Epstein-Barr virus in the pathogenesis of NPC," Seminars in Cancer Biology, vol. 12, no. 6, pp. 431-441, 2002.

[28] G. Henle and W. Henle, "Epstein Barr virus specific IgA serum antibodies as an outstanding feature of nasopharyngeal carcinoma," International Journal of Cancer, vol. 17, no. 1, pp. 1-7, 1976.

[29] M. Niens, A. Van Den Berg, A. Diepstra et al., "The human leukocyte antigen class I region is associated with EBV-positive Hodgkin's lymphoma: HLA-A and HLA complex group 9 are putative candidate genes," Cancer Epidemiology Biomarkers and Prevention, vol. 15, no. 11, pp. 2280-2284, 2006.

[30] K. A. McAulay, C. D. Higgins, K. F. Macsween et al., "HLA class I polymorphisms are associated with development of infectious mononucleosis upon primary EBV infection," Journal of Clinical Investigation, vol. 117, no. 10, pp. 3042-3048, 2007.

[31] S. Sengupta, J. A. Den Boon, I.-H. Chen et al., "Genomewide expression profiling reveals EBV-associated inhibition of MHC class I expression in nasopharynaeal carcinoma," Cancer Research, vol. 66, no. 16, pp. 7999-8006, 2006.

[32] F. Wiklund, "Prostate cancer genomics: can we distinguish between indolent and fatal disease using genetic markers?" Genome Medicine, vol. 2, no. 7, article 45, 2010.

[33] J. Bei, Y. Li, W. Jia et al., "A genome-wide association study of nasopharyngeal carcinoma identifies three new susceptibility loci," Nature Genetics, vol. 42, no. 7, pp. 599-603, 2010.

[34] M. Tang, J. A. Lautenberger, X. Gao et al., "The principal genetic determinants for nasopharyngeal carcinoma in China involve the HLA class I antigen recognition groove," PLoS Genetics, vol. 8, no. 11, Article ID e1003103, 2012. 

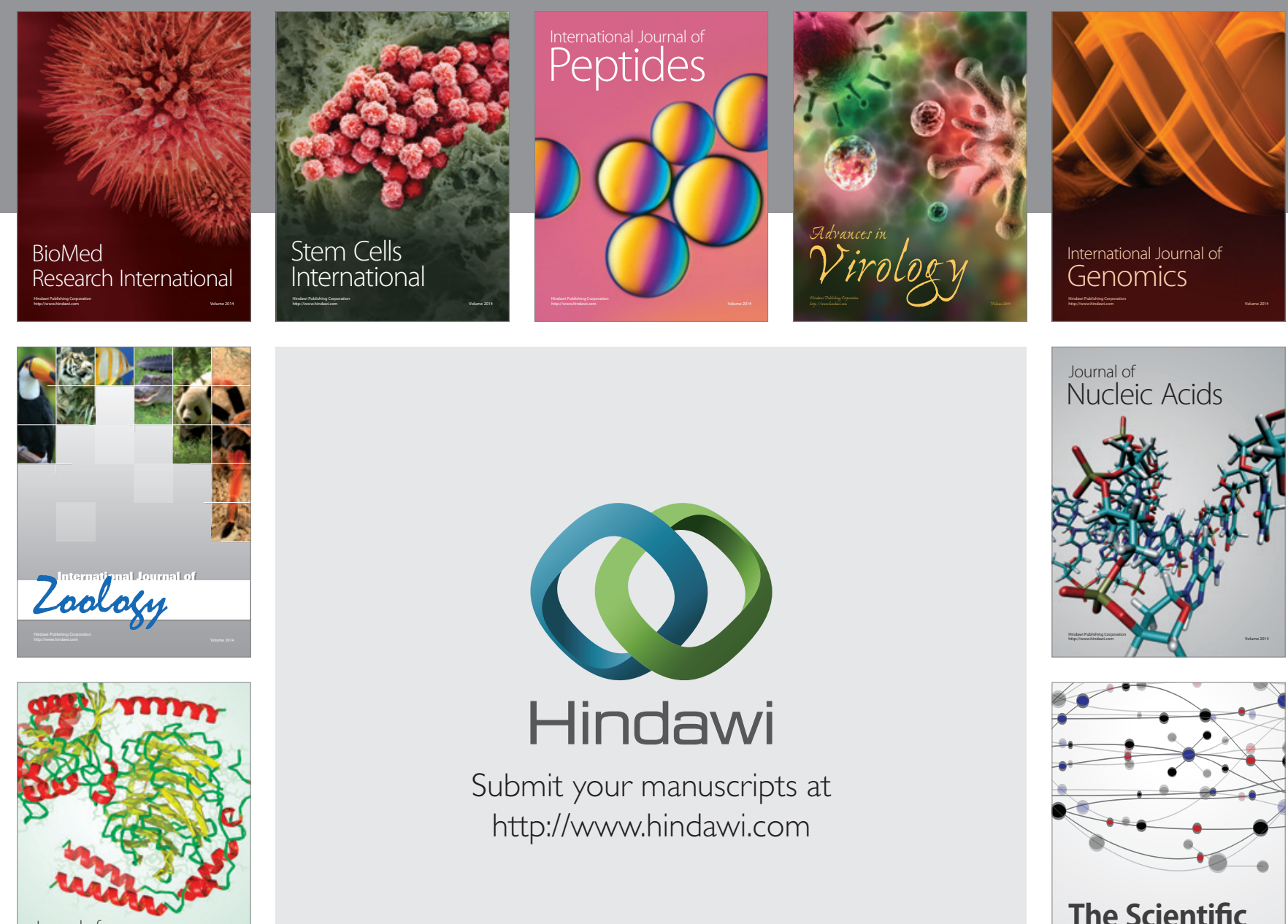

Submit your manuscripts at

http://www.hindawi.com

Journal of
Signal Transduction
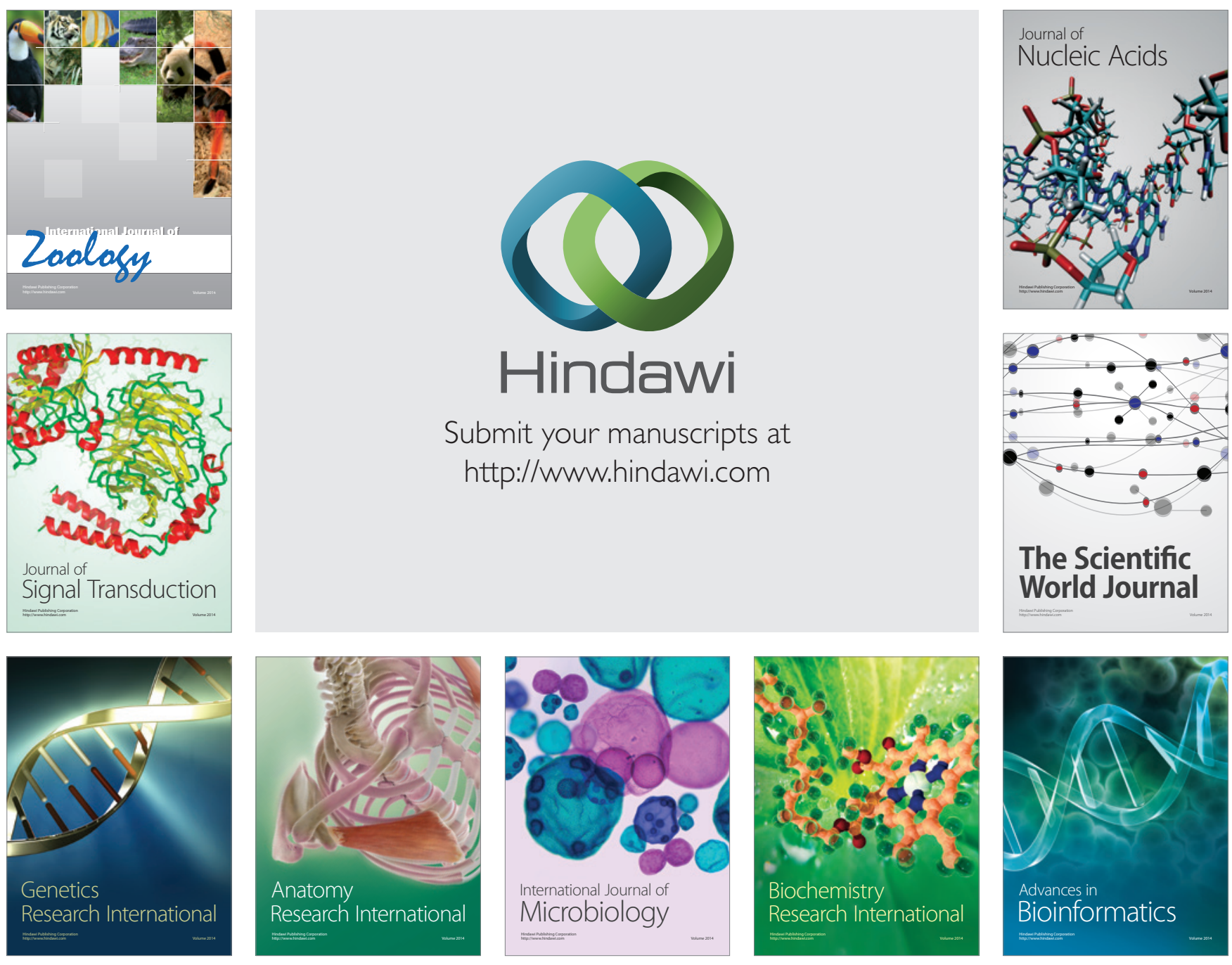

The Scientific World Journal
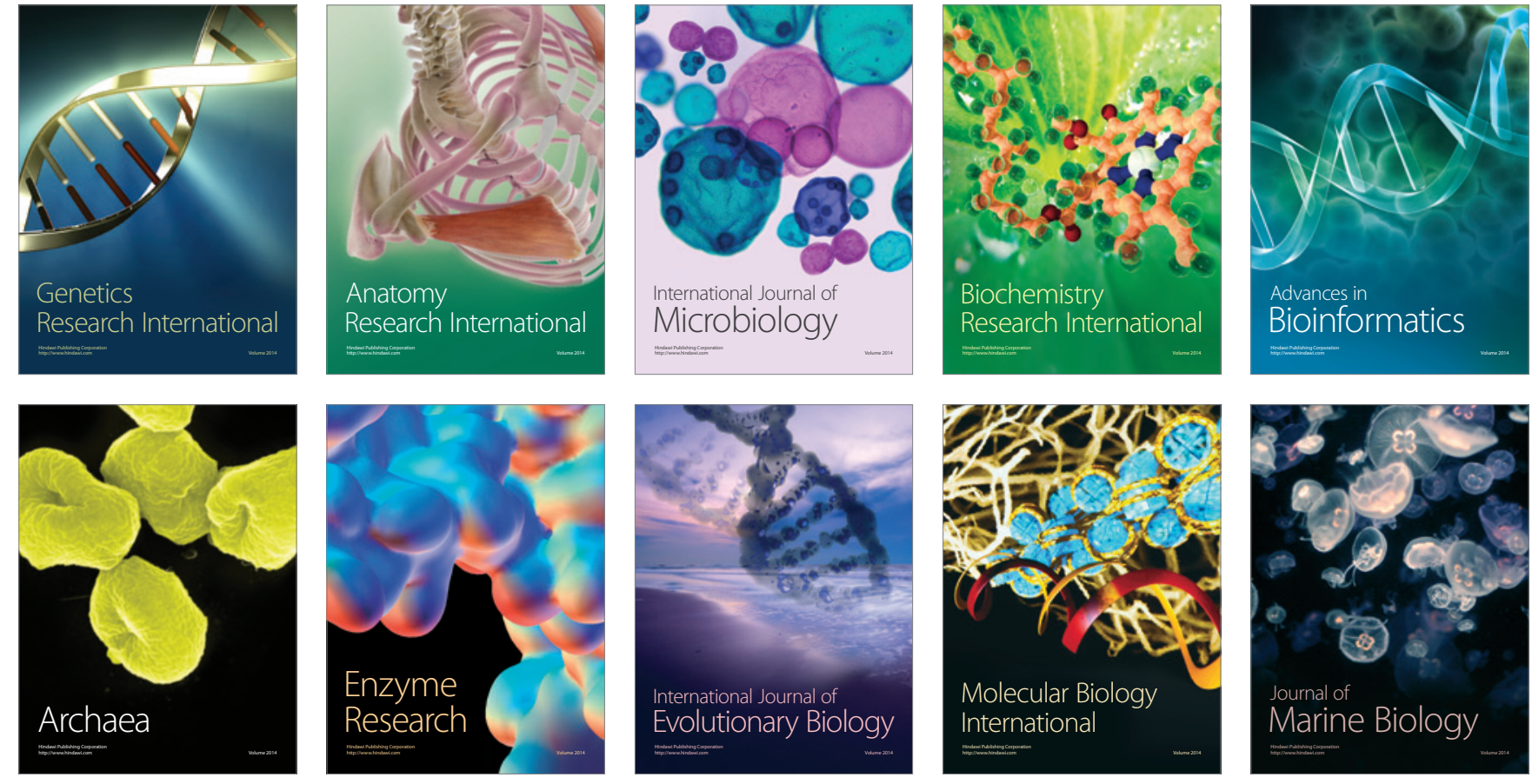